MATHEMATICS OF COMPUTATION

Volume 73, Number 246, Pages 987-1004

S 0025-5718(03)01529-1

Article electronically published on November 26, 2003

\title{
THE HOLOMORPHIC FLOW OF THE RIEMANN ZETA FUNCTION
}

\author{
KEVIN A. BROUGHAN AND A. ROSS BARNETT
}

\begin{abstract}
The flow of the Riemann zeta function, $\dot{s}=\zeta(s)$, is considered, and phase portraits are presented. Attention is given to the characterization of the flow lines in the neighborhood of the first 500 zeros on the critical line. All of these zeros are foci. The majority are sources, but in a small proportion of exceptional cases the zero is a sink. To produce these portraits, the zeta function was evaluated numerically to 12 decimal places, in the region of interest, using the Chebyshev method and using Mathematica.

The phase diagrams suggest new analytic properties of zeta, of which some are proved and others are given in the form of conjectures.
\end{abstract}

\section{INTRODUCTION}

The purpose of this paper is to contribute some insight into the nature of the Riemann zeta function $\zeta(s)$ through a study of its holomorphic flow,

$$
\dot{s}=\frac{d s}{d \tau}=\zeta(s)
$$

in the complex plane, where $s=\sigma+i t$ is a complex variable. Although the orbits or trajectories of the flow appear to give information only regarding the variation of $\arg \zeta(s)$, the flow characterizes the function [6]. This work is similar in spirit to that of Speiser [19], who based his conclusions on the behavior of $|\zeta(s)|$.

Following this introduction a brief description is given, with references, to the terms which are used in the paper from the field of continuous dynamical systems, intended as a summary for readers.

The production of the phase portraits of the flow presented a number of challenges, including the evaluation of $\zeta(s)$ to sufficient accuracy and with sufficient speed, and the development of suitable plotting techniques. It is not the purpose of this paper to report on this part of the work in detail. The computer systems Java, MATLAB, Mathematica and GP/Pari were used.

Although there are a reasonable number of figures in this paper, a very large number of additional diagrams were considered. (Readers who wish to consider the flow in the neighborhood of any of the first 500 zeros in more detail can gain access through the web site [7.)

Received by the editor April 7, 2002 and, in revised form, May 30, 2002

2000 Mathematics Subject Classification. Primary 30A99, 30C10, 30C15, 30D30, 32M25, 37F10, 37F 75 .

Key words and phrases. Dynamical system, phase portrait, critical point, orbit, separatrix, Riemann zeta function.

(C)2003 American Mathematical Society 
In section 2 a number of phase portraits are given. There are 3 overview plots: Figure 1 is the rectangle $[-7,3] \times[-30,30]$ showing many features but with minimal resolution; Figure 2 is the rectangle $[-10,10] \times[0,30]$ showing the first three zeros; Figure 3 is the region $[-5,4] \times[-4,4]$ including the pole and first sink and source on the $\sigma$-axis; then close-ups of the first $\operatorname{sink}(s=-2)$ in Figure 4 the first critical zero, Figure 5; and in Figure 6] the sink in the critical strip with the smallest $t$-coordinate. These diagrams are followed by a series of observations.

In section 3 the first 500 zeros are encoded to include information relating to their nature as source or sink, the direction of swirl and anisotropy in the flow near the zero. This provides at least a summary of the investigation. Although no obvious patterns or meaning is immediately apparent from this encoding, the appearance of just one sink with an anticlockwise swirl and positive NE-SW orientation is observed (near $t=650.669$ ).

Section 4 begins with some general theorems regarding the orbits, which can be derived in a straightforward manner. This is followed by the description (with proof) of the trivial zeros: they are alternately sinks and sources. Then, in the main part of the section, the nature of the zeros on the critical line is explored through a set of lemmas and theorems. For example, simple zeros off the critical line at mirror image points are of the same type. The flow in the neighborhood of any simple zero on the critical line is always that of a focus.

Section 5 describes some of the numerical analysis that underlies the creation of the phase portraits, including the choice of method and accuracy.

In the final section a set of conjectures about the Riemann zeta flow, relating to work in progress, is given.

1.1. Dynamical systems terminology. The equation $\dot{s}=\frac{d s}{d \tau}=\zeta(s)$ is considered, where $s$ is a complex variable and $\tau$ a real parameter interpreted as time. This corresponds to a system of two nonlinear ordinary differential equations (defined at each point of the complex plane other than $s=1)$, namely $\dot{\sigma}=\Re \zeta(\sigma+i t)$ and $\dot{t}=\Im \zeta(\sigma+i t)$. Since the right-hand side of this system does not depend on $\tau$, the system is called autonomous. Through each point $s_{o}$ there is a unique solution $\gamma\left(s_{o}, \tau\right)$, with $\gamma\left(s_{o}, 0\right)=s_{o}$, which will exist on an open interval of values of the parameter $\tau$ called the maximal domain of existence, which may be the whole of $\mathbb{R}$. Regarded as a mapping $\tau \rightarrow \gamma\left(s_{o}, \tau\right)$, this solution is called an orbit or trajectory. The ensemble of orbits is called a holomorphic flow. A graphical representation of a subset of the orbits is called a phase portrait or phase diagram.

In any study of a particular flow the nature of the orbits in the neighborhood of each zero of the right-hand side is of particular interest. The zeros are called singular or critical or equilibrium points. For functions with reasonable regularity (two continuous derivatives is enough) then the flow near a zero corresponds to the flow produced by the linearization of the equations at that point: in the case of $\zeta(s)$, the linearized system near the zero $\rho$ has the form $\dot{s}=\zeta^{\prime}(\rho)(s-\rho)$.

A saddle point is a zero for which there are trajectories which tend to the point in both positive and negative time. A center is a zero which has a neighborhood such that every solution curve in the neighborhood, other than the zero itself, is a closed curve with the zero in its interior. A zero is called stable or a sink (unstable or a source) if there is a neighborhood such that every solution curve with initial point in the neighborhood tends to the zero in positive (negative) time tending to $\infty(-\infty)$. A zero is a node if there is a neighborhood such that every orbit in the 
neighborhood tends to the zero with a well defined tangent in either positive or negative time. It is a focus if every orbit in a neighborhood tends to the zero and circulates about the zero an infinite number of times while doing so.

The basin of attraction of a stable zero is the set of all points such that the orbit through each point tends to the zero as $\tau \rightarrow \infty$. The basin of repulsion of an unstable zero is the set of all points such that the orbit through each point tends to the zero as $\tau \rightarrow-\infty$.

Periodic orbits are trajectories which come back to the initial (or any) point after a finite time interval. Limit cycles are isolated periodic orbits, in that they are periodic and have a neighborhood containing no other periodic orbits.

A separatrix is normally defined to be either a zero, a limit cycle or a trajectory which lies on the boundary of what is known as a hyperbolic sector. Since the holomorphic flows considered do not have hyperbolic sectors (other than by an extension of the definition at the single pole of $\zeta(s)$ or possibly at the point of infinity - and we do not employ a compactification here), a separatrix is defined to be any trajectory on the boundary of the basin of attraction or repulsion of a sink or a source.

The types of zeros of holomorphic flows are restricted to being centers, nodes, or foci when the zeros are simple, and to having all elliptic sectors when the the zeros are of order higher than one, 6].

A good introduction to the dynamical systems used here is [18] or [8]. Some of the results from the paper by the first author [6] are referenced.

\section{Phase portraits}

Examples of phase portraits are given below. Some of the observations made below arise from these and other images considered by the authors. Comments are restricted to the upper half plane and the $\sigma$-axis. The lower half plane has mirror image orbits (Figure 1). The comments are restricted to the parts of the plane which have been investigated, including the first 500 critical zeros.

1. The $\sigma$-axis: The $\sigma$-axis is an orbit, since $\zeta(s)$ is real there. To the right of the pole at $s=1$ (Figure 3) the orbit points to the right and between the pole and the sink at $s=-2$ to the left. The right-left alternation continues moving along the axis towards the left.

2. Basins of the trivial zeros: Each trivial zero is a node with the first being a sink and then alternating sources and sinks. The zeros at -2 (sink) and -4 (source) are special (Figure 2), whereas the sinks at $-6,-10,-14, \ldots$ and sources at $-8,-12,-16, \ldots$ are in a regular pattern.

Taking the latter first, every regular orbit from a source ends up at one of the neighboring sinks. Between the orbits which go to the left sink and those which go to the right sink there is a separatrix. These separatrices tend to infinity in a NW direction.

As for the source at -4 , some of the orbits tend to the sink at -6 , some cross the line $\sigma=1$ and join the horizontal orbits going to infinity in the $\sigma$-direction, and some go to the sink at $s=-2$. One separatrix tends to infinity in the NW direction, like other trivial sources. Another separates the orbits from those of the first critical zero, and a third goes to the pole.

3. The pole: The orbits (Figure 3) look like those of a saddle point, although the behavior of course is different since $s=1$ is not a zero. One pair of separatrices 


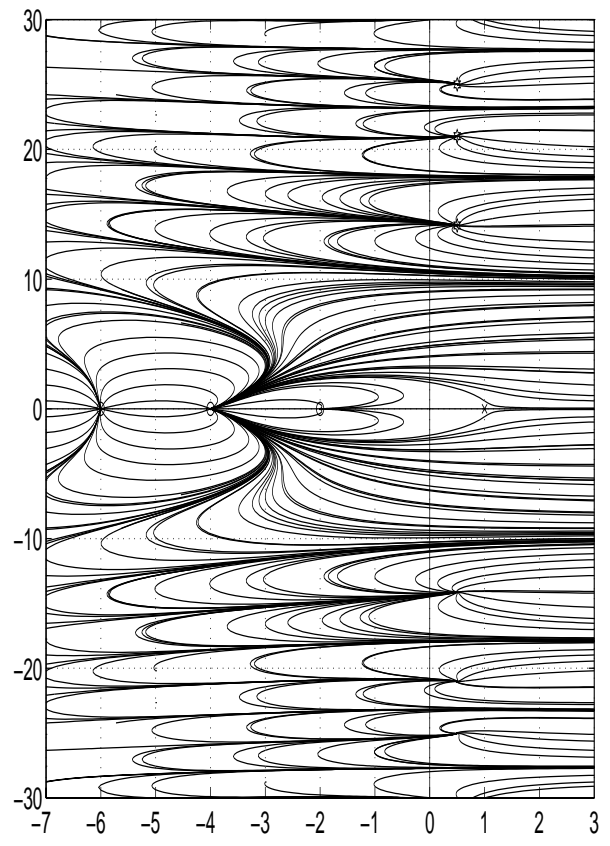

Figure 1. Overview of the orbits for $\dot{s}=\zeta(s)$ on $[-7,3] \times[-30,30]$

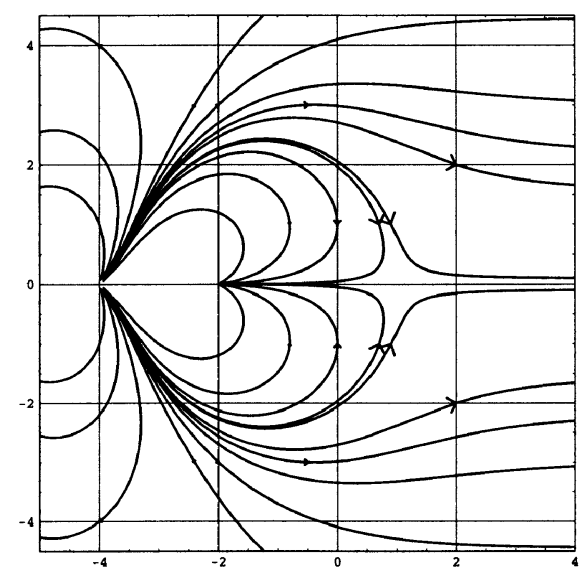

FiguRE 3. Region near the pole at $s=1$

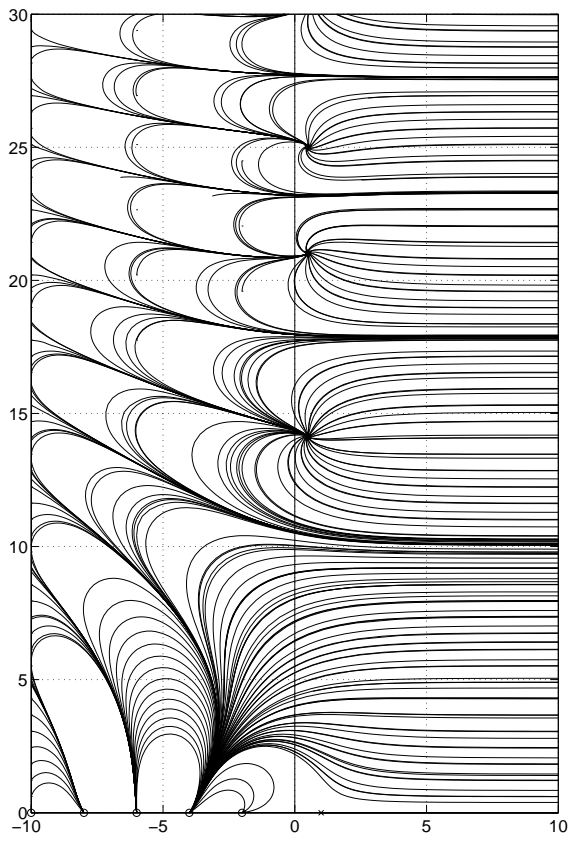

Figure 2. The lower section of the strip $[-10,10] \times[0,30]$

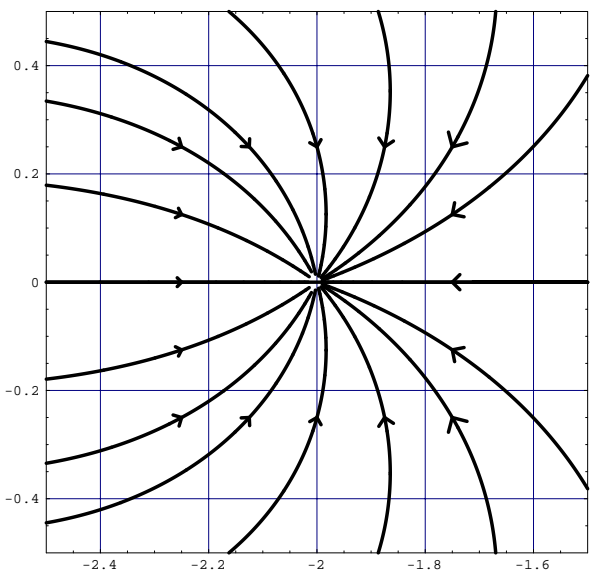

Figure 4. Region about the sink $s=-2$

lies in the $\sigma$-axis; the other pair is tangent to the line $\sigma=1$. Orbits to the NW of $s=1$ go from the source at $s=-4$ and to the sink at $s=-2$. Orbits to the NE start at $s=-4$ and go to infinity in the $\sigma$-direction. One separatrix comes from the source at $s=-4$. The other two lie in the $\sigma$-axis and go to positive infinity or to the sink at $s=-2$. 


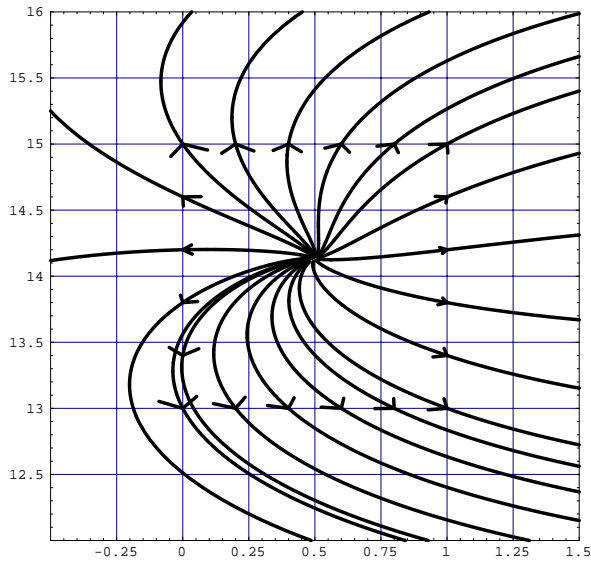

Figure 5. The first critical zero, near $s=0.5+$ $14.1347 i$, zero no. 1

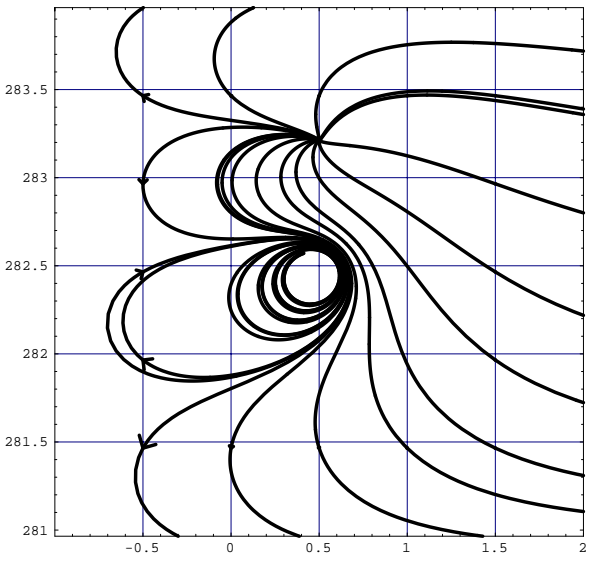

Figure 6 . The first critical sink, near $s=0.5+$ $282.465 i$, zero no. 127

4. Basins of the critical zeros: Each critical zero, apart from 13 exceptions (zeros number $127,136,196,213,233,256,289,368,379,380,399,401$, and 462), is an unstable focus (source). The exceptional zeros are stable and are sinks. A number of zeros come very close to being centers, and a number to being nodes. Separatrices for the zeros which are sources are of two types: those that start at infinity in the NW direction and end up asymptotically approaching horizontal lines (these run between each of the critical zeros), and those that start at the zero and tend to infinity in a NW direction (these divide the orbits associated with the zero that start left, then head back towards the right and above the zero or left and below the zero). See Figure 2.

In the case of the 13 exceptional zeros there is just one separatrix which loops around the zero coming from infinity and tending to it in a NW direction.

5 . Behavior of $\zeta$ on the line $s=1+i t$ : The general direction of the orbits as they cross the line $\sigma=1$ is left-to-right. However, this is reversed in the neighborhood of the zeros which are sinks.

6 . To the right of the line $\sigma=1$ the vectors generally point to the right. By say $\sigma=9$ they are very close to $\zeta(s)=1$; indeed, for $\Re(s) \geq 10,|\zeta(s)-1|<10^{-3}$.

7. To the left of the line $\sigma=0$ the orbits are approximately those of the equation

$$
\frac{d s}{d \tau}=2(2 \pi)^{s-1} \Gamma(1-s) \sin \left(\frac{1}{2} \pi s\right),
$$

with the approximation improving as $\sigma$ becomes larger and more negative.

\section{Symbolic Patterns of the Zeros}

Theorem 3.1. Let

$$
\dot{z}=(\alpha+i \beta) z+(a+i b) z^{2}=f(z)
$$

be a polynomial holomorphic vector field with $\alpha, \beta, a, b$ real and nonzero. Then the flow has an anisotropic focus at $z=0$ with the principal direction (defined as the direction in which the trajectories in a neighborhood of 0 have maximum curvature) 
given by

$$
\tan (\theta)=\frac{a\left(2 \alpha^{2}+\beta^{2}\right)+b \alpha \beta}{b\left(2 \alpha^{2}+\beta^{2}\right)-a \alpha \beta} .
$$

Proof. Let $f(z)=u+i v$. The square of the curvature of the flow may be written [6] as

$$
\kappa^{2}=\frac{v_{x}^{2}}{u^{2}+v^{2}} .
$$

Converting to polar coordinates, we obtain

$$
\kappa^{2}=\frac{\beta^{2}+4 b \beta r c+4 b^{2} r^{2} c^{2}+4 a \beta r s+8 a b r^{2} c s+4 a^{2} r^{2} s^{2}}{\alpha^{2}+\beta^{2}+\left(a^{2}+b^{2}\right) r^{2}+2 r((a \alpha+b \beta) c+(-\alpha b+a \beta) s)},
$$

where $c=\cos (\theta)$ and $s=\sin (\theta)$.

To derive the given expression for $\tan (\theta)$, partially differentiate this expression with respect to $\theta$ and equate the coefficient of the term of highest order in $r$ (i.e., $\left.1 / r^{2}\right)$ as $r \rightarrow 0+$ to zero, to obtain the direction of maximum curvature,

The following encoding of the first 500 zeros is considered. A zero of $\zeta(s)=u+i v$ is encoded by one of the 8 letters $\mathrm{A}$ through $\mathrm{H}$ depending on the signs of $u_{x}, v_{y}$ and $\tan (\theta)$ as set out in Table 1. These parameters determine whether the zero is a source or sink, has positive or negative swirl and is anisotropic in a NE-SW or NW-SE direction, respectively.

The first 500 critical zeros have the encoding shown in Table2,

To obtain this representation, Odlyzko's 8 decimal place zeros [16] were used with Mathematica to evaluate the first and second derivatives of the zeta function. These

TABLE 1. Encoding the zeta zeros

\begin{tabular}{|c|c|c|c|r|}
\hline Letter code & $\alpha$ & $\beta$ & $\tan (\theta)$ & occurrences \\
\hline $\mathrm{A}$ & + & + & + & 214 \\
\hline $\mathrm{B}$ & + & + & - & 37 \\
\hline $\mathrm{C}$ & + & - & + & 32 \\
\hline $\mathrm{D}$ & + & - & - & 204 \\
\hline $\mathrm{E}$ & - & + & + & 1 \\
\hline $\mathrm{F}$ & - & + & - & 4 \\
\hline $\mathrm{G}$ & - & - & + & 7 \\
\hline $\mathrm{H}$ & - & - & - & 1 \\
\hline
\end{tabular}

TABLE 2. Encoding of the first 500 critical zeros

\begin{tabular}{l} 
ADADADDADAADDADAAADAADADADDAADDAACAAADDBADDADADDAA \\
DADAADDAAADDDBDDABADCAAADADBADDDADCDDAADDADADDDAAD \\
CDABACCAAADADBBDDAABADDABBGAADAADDDFADDADAACDAAADA \\
DAAADDAADBDDDABDDADAAACDAAADDDDABCCDBBAADDAAAGCADA \\
ADDCBBDDDDADFDDAAADADDBADAADAGADAADDADDADDABDA \\
DADAAFCDADADADCAAADDDADAADDDAAAADAAAHABADADDDCAB \\
DDDAABAADDABADCBDAAADDDAADADDDDAACDAADADADDAAADDDA \\
DABDDCDBADDACAAADGAABAADDDABEGCDAABAADDDBAACAADDFA \\
GCDAADDADAAABDDDADAADACCAADDDADADBDCABAADCCDDAADDD \\
DBCBDDDADAAGDADAAAADDAAADDCADDABCCDAAADDADDAAACDAA \\
\hline
\end{tabular}


evaluations were confirmed with MATLAB. The following patterns are observed: $\mathrm{A}$ and $\mathrm{D}$ repeat, and the repeating blocks alternate, other than the occasional $\mathrm{C}$ and $\mathrm{B}$, which also might repeat. $\mathrm{F}$ and $\mathrm{G}$ are rare, and $\mathrm{E}$ or $\mathrm{H}$ even rarer. The single occurrence of $\mathrm{E}$ is at zero number 379 , near 650.669 , and that of $\mathrm{H}$ at zero number 289 , near 527.904 .

The data used to derive this encoding is given on the website 7 .

\section{THEOREMS}

\subsection{General results about orbits.}

Theorem 4.1. 1. The flow is the transpose of a gradient field. In every simply connected subregion $\Omega$ of $\mathbb{C}$ which does not include $s=1$, there is an harmonic function $F(x, y)$, unique up to an additive constant, such that if $\zeta(s)=u+i v$, then $u=F_{y}$ and $v=F_{x}$.

2. The real axis consists of orbits separated by the pole at $s=1$ and zeros at $s=-2 n, n \in \mathbb{N}$.

3. The orbits in the lower half plane are the mirror image of the orbits in the upper half plane.

4. For $\Re s \geq \delta$, where $\delta$ is the solution to $\zeta(\sigma)=2$ in $(1,2)$, every orbit points strictly to the right.

5. In every strip $1 \leq \Re s \leq 1+\epsilon, \epsilon>0$, for every given direction, there are an infinite number of orbits which point in that direction.

6. In a (deleted) neighborhood of the pole at $s=1$ the orbits are those of a saddle point for the flow $\dot{s}=\zeta(s)|\zeta(s)|^{-2}$.

7. The separatrices for the pole at $s=1$ lie along the axis $t=0$ and lines tangent to the line $\sigma=1$.

8. If 凡s is sufficiently large and negative, then the argument of $\zeta(s)$ at $s=\sigma+i t$ is given approximately by $f(\sigma, t) \bmod 2 \pi$, where

$f(\sigma, t)=(1+\log (2 \pi)) t-\frac{1}{2} t \log \left((1-\sigma)^{2}+t^{2}\right)+\left(\frac{1}{2}-\sigma\right) \arctan \left(\frac{t}{\sigma-1}\right)+\frac{\pi}{2}(1-\sigma)$.

Proof. 1. Fix a point $a \in \Omega$ and for each $x+i y \in \Omega$ let

$$
F(x, y)=\int_{a}^{x+i y} v d x+u d y
$$

where the path of integration $\Gamma$ is any rectifiable curve lying entirely in $\Omega$. Because $F$ is the imaginary part of $\int_{\Gamma} \zeta(s) d s$, it is harmonic. It follows directly from the definition of $F$ that $F_{y}=u$ and $F_{x}=v$.

2. Since $\zeta$ is real on the real axis, this is an orbit where $\zeta$ is defined and nonzero.

3. If $\gamma(\tau)$ is an orbit, then

$$
\overline{\gamma(\tau)}=\overline{\gamma(\tau)}=\overline{\zeta(\gamma(\tau))}=\zeta(\overline{\gamma(\tau)})
$$

so $\overline{\gamma(\tau)}$ is an orbit also.

4. Let $\delta=1.7286472389981 \cdots$ be the solution to the equation $2=\zeta(\sigma)$ in the real interval $(1,2)$. Then, for $s=\sigma+i t$ with $\sigma$ in this interval,

$$
\begin{aligned}
\Re \zeta(s) & =1+\frac{\cos (t \log 2)}{2^{\sigma}}+\frac{\cos (t \log 3)}{3^{\sigma}}+\cdots \\
& \geq 1-\frac{1}{2^{\sigma}}-\frac{1}{3^{\sigma}}-\cdots \\
& =2-\zeta(\sigma)>0
\end{aligned}
$$


provided $\delta<\sigma$, since $\zeta(\sigma)$ is monotonically decreasing on $(1,2)$.

If $\Re \zeta(\delta+i t)=0$ for some $t \neq 0$, then

$$
0=1+\frac{\cos (t \log 2)}{2^{\delta}}+\frac{\cos (t \log 3)}{3^{\delta}}+\cdots,
$$

and

$$
2=1+\frac{1}{2^{\delta}}+\frac{1}{3^{\delta}}+\cdots
$$

So

$$
0=\frac{1+\cos (t \log 2)}{2^{\delta}}+\frac{1+\cos (t \log 3)}{3^{\delta}}+\cdots .
$$

Since each term in the sum on the right is positive and the sum is absolutely convergent, each term vanishes. This means, for example, that $t \log 2$ and $t \log 3$ are both odd integral multiples of $\pi$, which is impossible. Hence for all $t, \Re \zeta(\delta+i t) \neq 0$.

5 . This is a direct consequence of a (small variation of a) result of Bohr (see [20]), that in every strip with $1 \leq \sigma \leq 1+\epsilon, \zeta(s)$ takes on every value except zero an infinite number of times.

6. That the flow lines are those of a saddle is a special case of a proposition in [6]. Their identity with those of the given flow, which is nonholomorphic, but has a zero at $s=1$, is also immediate.

7. Consider the flow $\dot{s}=1 / \zeta(\bar{s})$, which has a zero at $s=1$ and is analytic in the neighborhood $B(1,1)$ of $s=1$. The stable manifold lies in the $\sigma$-axis, and the unstable manifold, which is at right angles to it, must therefore be tangent to the line $\sigma=1$.

8. Since for these values of $\sigma$ we have $\zeta(1-s) \approx 1$, the functional equation gives

$$
\frac{d s}{d \tau}=2(2 \pi)^{s-1} \Gamma(1-s) \sin \left(\frac{1}{2} \pi s\right) .
$$

The given expression for the argument is derived using Stirling's approximation for the logarithm of the gamma function and setting

$$
\arctan \frac{\tanh (t \pi / 2)}{\tan (\sigma \pi / 2)}=\frac{\pi}{2}-\frac{\pi \sigma}{2},
$$

in the above approximation to the zeta function flow.

Notes. (i) It can be observed from the phase portraits that the real part of $\zeta(1+i t)$ is positive for every $t$ with $0<t<812$, and this phenomenon appears to persist through much larger values of $t$, appearing to contradict statement 5 of the above theorem. The existence of sinks distorting the flow lines (see Figure 8 ) appears to result in kinks which could explain 5 .

(ii) It is expected that there is a zero for the real part of $\zeta(s)$ on the line $\sigma=\eta$ for $1 \leq \eta<\delta$. However, phenomenologically, the predominant direction of the field remains to the right in this range. For example, it follows from a careful reading of the proof of the classical theorem based on the $n$-dimensional Dirichlet box principle (for example, see [20], Theorem 8.4) that for every $\epsilon>0, \Re \zeta(s) \geq(1-\epsilon) \zeta(\sigma)$ for indefinitely large values of $t$.

When $t$ is in the range $[0,1000]$, then $\Re \zeta(s) \geq 0.5$ on $\sigma=\delta$.

(iii) The expression in 8 is close to being linear in $t$ for fixed $\sigma$ and reproduces the oscillation in direction of $\dot{s}=\zeta(s)$ (see Figure 17) very accurately, even at a value of $\sigma$ as small as $\sigma=-4$. 


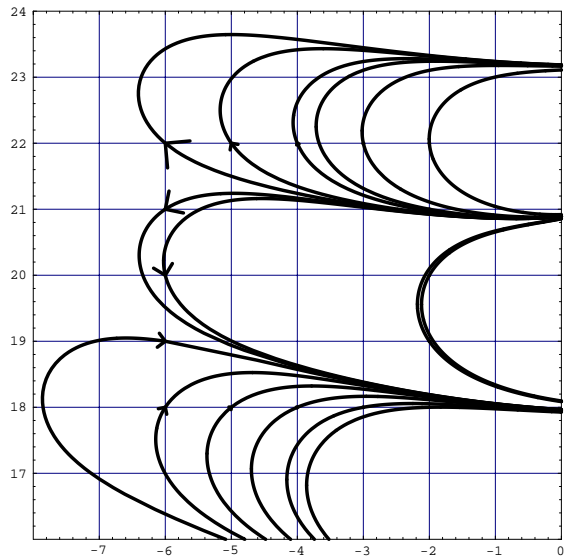

Figure 7. Subregion of the upper left half plane $[-8,0] \times[16,24]$

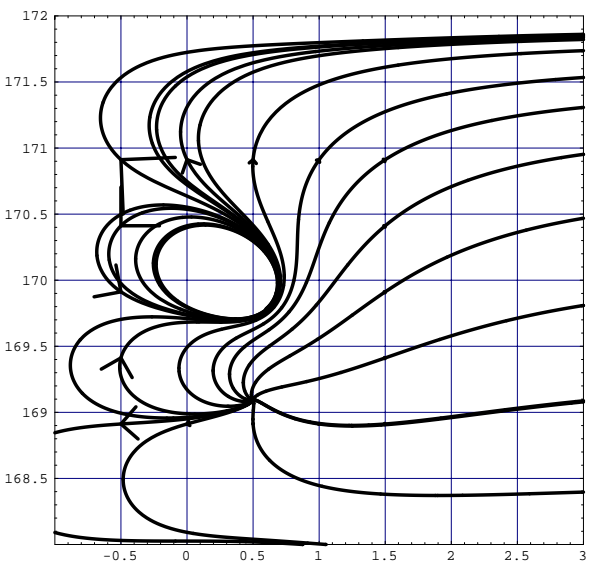

Figure 8. The source near $s=0.5+169.912 i$ behaving like a sink

\subsection{Nature of the trivial zeros.}

Theorem 4.2. The trivial zeros occurring at $s=-2 n, n=1,2, \ldots$, are nodes, being alternately sources and sinks, the node at $s=-2$ being a sink.

Proof. Since $\zeta(s)$ is real on the $\sigma$-axis, $v=v_{\sigma}=0$; so the derivative, where it exists, is also real. Hence [6] each simple zero is a node. These are at $s=-2 n, n=1,2, \ldots$, where

$$
\zeta^{\prime}(-2 n)=(-1)^{n} \frac{n(2 n-1) !}{(2 \pi)^{2 n}} \zeta(2 n+1),
$$

so the derivative is real and the sign alternates. Therefore the trivial zeros are nodes, and alternately sinks and sources for $n=1,2, \ldots$.

\subsection{Nature of the zeros on the critical line.}

Theorem 4.3. If $0<x<\frac{1}{2}, y>2 \pi, s=\frac{1}{2}+x+i y$ and $\zeta(s) \neq 0$, then the magnitude of $\zeta$ at $s$ is, asymptotically in $y$, never the same as its magnitude at its mirror image point in $\sigma=\frac{1}{2}$, namely $\frac{1}{2}-x+i y$. Indeed, for all $\epsilon>0$ there is a $y_{\epsilon}>0$ such that the ratio of the two magnitudes satisfies

$$
\frac{\left|\zeta\left(\frac{1}{2}-x+i y\right)\right|}{\left|\zeta\left(\frac{1}{2}+x+i y\right)\right|} \geq(1-\epsilon) e^{x \log \left(\frac{y}{2 \pi}\right)}
$$

for $y>y_{\epsilon}$.

Proof. Let

$$
\gamma(s)=\pi^{-s / 2} \Gamma\left(\frac{s}{2}\right) \zeta(s), \quad s \neq 0,1
$$

Let

$$
s=\frac{1}{2}+x+i y, \quad 0<y, 0<x<\frac{1}{2},
$$

and let $\epsilon>0$ be given. 
Then, since $\gamma(s)=\gamma(1-s)$,

$$
\begin{aligned}
& \pi^{-\left(\frac{1}{2}+x+i y\right) / 2} \Gamma\left(\left(\frac{1}{2}+x+i y\right) / 2\right) \zeta\left(\frac{1}{2}+x+i y\right) \\
& =\pi^{-\left(\frac{1}{2}-x-i y\right) / 2} \Gamma\left(\left(\frac{1}{2}-x-i y\right) / 2\right) \zeta\left(\frac{1}{2}-x-i y\right) .
\end{aligned}
$$

Let

$$
f(x, y)=\frac{\left|\zeta\left(\frac{1}{2}-x+i y\right)\right|}{\left|\zeta\left(\frac{1}{2}+x+i y\right)\right|}
$$

Then, by equation (1) above,

$$
f(x, y)=\pi^{-x} \frac{\left|\Gamma\left(\frac{1}{4}+x / 2+i y / 2\right)\right|}{\left|\Gamma\left(\frac{1}{4}-x / 2+i y / 2\right)\right|} .
$$

Stirling's approximation gives, for $\Re s>0$,

$$
\Gamma(s)=e^{-s} s^{s-\frac{1}{2}} \sqrt{2 \pi}\left(1+O\left(\frac{1}{s}\right)\right) .
$$

So, considering $y$ sufficiently large $\left(y \geq y_{o}\right)$ and discarding terms of order $1 / y$, $f(x, y)$ may be written as

$$
\pi^{-x} e^{-x}\left|\frac{\left(\frac{1+2 x}{4}+\frac{i y}{2}\right)^{-\frac{1-2 x}{4}}}{\left(\frac{1-2 x}{4}+\frac{i y}{2}\right)^{-\frac{1+2 x}{4}}} \times \frac{\left(\frac{1+2 x}{4}+\frac{i y}{2}\right)^{\frac{i y}{2}}}{\left(\frac{1-2 x}{4}+\frac{i y}{2}\right)^{\frac{i y}{2}}}\right|,
$$

which simplifies to

$$
e^{-(1+\log \pi) x} \frac{\left(\left(\frac{1+2 x}{4}\right)^{2}+\left(\frac{y}{2}\right)^{2}\right)^{-\frac{1-2 x}{8}}}{\left(\left(\frac{1-2 x}{4}\right)^{2}+\left(\frac{y}{2}\right)^{2}\right)^{-\frac{1+2 x}{8}}} \times \frac{e^{-\frac{y}{2} \arctan \frac{2 y}{1+2 x}}}{e^{-\frac{y}{2} \arctan \frac{2 y}{1-2 x}}},
$$

where the principal value of the arctan function has been taken, the angles being in the range $(0, \pi / 2)$.

Therefore $f(x, y)=A \times B$, where

$$
\begin{aligned}
A & =\exp \left(-(1+\log \pi) x+\frac{y}{2}\left(\arctan \frac{2 y}{1-2 x}-\arctan \frac{2 y}{1+2 x}\right)\right), \\
B & =\left[\frac{\left(\left(\frac{1+2 x}{4}\right)^{2}+\left(\frac{y}{2}\right)^{2}\right)}{\left(\left(\frac{1-2 x}{4}\right)^{2}+\left(\frac{y}{2}\right)^{2}\right)}\right]^{\frac{1}{8}} \times\left(\left(\left(\frac{1-2 x}{4}\right)^{2}+\left(\frac{y}{2}\right)^{2}\right)\left(\left(\frac{1+2 x}{4}\right)^{2}+\left(\frac{y}{2}\right)^{2}\right)\right)^{\frac{x}{4}} \\
& =C \times D .
\end{aligned}
$$

Now consider

$$
\frac{f(x, y)}{e^{x \log \frac{y}{2 \pi}}}=\frac{A}{e^{-x \log \pi}} \times C \times \frac{D}{\left(\left(\frac{y}{2}\right)^{2}\right)^{\frac{x}{4}}\left(\left(\frac{y}{2}\right)^{2}\right)^{\frac{x}{4}}} .
$$

First,

$$
\begin{aligned}
\frac{A}{e^{-x \log \pi}} & =\exp \left(-x+\frac{y}{2}\left(\arctan \frac{2 y}{1-2 x}-\arctan \frac{2 y}{1+2 x}\right)\right) \\
& =\exp \left(-\frac{3 x+4 x^{3}}{12 y^{2}}+O\left(\frac{1}{y^{3}}\right)\right) \\
& \geq 1-\epsilon / 2, \quad \text { for } y \geq y_{1} .
\end{aligned}
$$

Also,

$$
C=1-\frac{x}{4} \frac{1}{y^{2}}+O\left(\frac{1}{y^{4}}\right) \geq 1-\epsilon / 2
$$


for $y \geq y_{2}$. Finally,

$$
\begin{aligned}
\frac{D}{\left(\left(\frac{y}{2}\right)^{2}\right)^{\frac{x}{4}}\left(\left(\frac{y}{2}\right)^{2}\right)^{\frac{x}{4}}} & =\left[\left(1+\left(\frac{1-2 x}{2 y}\right)^{2}\right)\left(1+\left(\frac{1+2 x}{2 y}\right)^{2}\right)\right]^{\frac{x}{4}} \\
& =1+\frac{x\left(1+4 x^{2}\right)}{8 y^{2}}+O\left(\frac{1}{y^{4}}\right) \\
& \geq 1
\end{aligned}
$$

for $y \geq y_{3}$. Hence, for $y \geq y_{\epsilon}=\max \left\{y_{o}, y_{1}, y_{2}, y_{3}\right\}$,

$$
f(x, y) \geq(1-\epsilon) e^{x \log \frac{y}{2 \pi}} .
$$

Lemma 4.1. The sum $\arg \Gamma\left(\left(\frac{1}{2}+x+i y\right) / 2\right)+\arg \Gamma\left(\left(\frac{1}{2}-x+i y\right) / 2\right)$ is asymptotically constant for $x \in\left[0, \frac{1}{2}\right]$. In particular, its derivative with respect to $x$ is $O(1 / y)$ as $y \rightarrow \infty$, where the implied constant is absolute.

Proof. Let

$$
g(x, y)=\arg \Gamma\left(\left(\frac{1}{2}+x+i y\right) / 2\right)+\arg \Gamma\left(\left(\frac{1}{2}-x+i y\right) / 2\right) .
$$

Then

$$
\frac{\partial g(x, y)}{\partial x}=-\frac{x}{y}+\frac{x\left(4 x^{2}-9\right)}{12} \frac{1}{y^{3}}+O\left(\frac{1}{y^{4}}\right)
$$

as $y \rightarrow \infty$.

Lemma 4.2. Let the entire function $\Lambda$ be defined by

$$
\Lambda(s)=2(2 \pi)^{-s} \Gamma(s) \cos \left(\frac{1}{2} \pi s\right) .
$$

Then for all $s, \Lambda\left(P_{-}\right) \overline{\Lambda\left(P_{+}\right)}=1$, where $P_{+}=\frac{1}{2}+x+i y$ and $P_{-}=\frac{1}{2}-x+i y$.

Proof. The functional equation for $\zeta(s)$ may be written $\zeta(1-s)=\Lambda(s) \zeta(s)$. If $P_{+}$ is not a zero of $\zeta$, then

$$
\begin{aligned}
& \overline{\zeta\left(P_{-}\right)}=\Lambda\left(P_{+}\right) \zeta\left(P_{+}\right), \\
& \overline{\zeta\left(P_{+}\right)}=\Lambda\left(P_{-}\right) \zeta\left(P_{-}\right) .
\end{aligned}
$$

Hence $\overline{\zeta\left(P_{+}\right)}=\Lambda\left(P_{-}\right) \overline{\Lambda\left(P_{+}\right) \zeta\left(P_{+}\right)}$, and so $1=\Lambda\left(P_{-}\right) \overline{\Lambda\left(P_{+}\right)}$. If $P_{+}$is a zeta zero, then the result follows by continuity, since each zero is isolated.

Lemma 4.3. With the same notion as that used in Lemma 4.2 above, the difference

$$
\arg \Gamma\left(P_{-}\right)-\arg \Gamma\left(P_{+}\right) \equiv \pi x+\frac{2 \sin (\pi x)}{e^{2 \pi y}}+O\left(\frac{1}{e^{4 \pi y}}\right) \quad \bmod 2 \pi
$$

as $y \rightarrow \infty$, where the implied constant is absolute.

Proof. Let $s=\frac{1}{2}+x+i y$ in

Then

$$
\Gamma(s) \Gamma(1-s)=\frac{\pi}{\sin \pi s} .
$$

$$
\arg P_{+}-\arg P_{-} \equiv \arg \cos (\pi x+i \pi y) \quad \bmod 2 \pi
$$

leading to

$$
\arg P_{-}-\arg P_{+} \equiv \arctan [\tan \pi x \tanh \pi y] \quad \bmod 2 \pi,
$$

and from this expression the given asymptotic form follows directly. 
Theorem 4.4. The sum $\arg \zeta\left(\frac{1}{2}+x+i y\right)+\arg \zeta\left(\frac{1}{2}-x+i y\right)$ is asymptotically constant for $x \in\left(0, \frac{1}{2}\right)$. In particular, its derivative with respect to $x$ is $O(1 / y)$ as $y \rightarrow \infty$, where the implied constant is absolute.

Proof. Take the argument of each side of equation (1) of Theorem 4.3 This gives

$$
\begin{aligned}
& -y+\arg \Gamma\left(\left(\frac{1}{2}+x+i y\right) / 2\right)+\arg \Gamma\left(\left(\frac{1}{2}-x+i y\right) / 2\right) \\
& \quad \equiv-\arg \zeta\left(P_{+}\right)-\arg \zeta\left(P_{-}\right) \bmod 2 \pi .
\end{aligned}
$$

Differentiate partially with respect to $x$. The result of the theorem then follows from Lemma 4.1 above.

Theorem 4.5. Let $\zeta(s)$ have a simple zero at $s=\frac{1}{2}+x+i y$ for some $x, y>0$. Then the zero at the reflected point $\frac{1}{2}-x+i y$ is also simple and has the same local type, i.e., both are centers, nodes or foci. If they are centers or foci, then both have the same direction of swirl. If they are nodes or foci, then both are either sources or sinks.

Proof. The same notation is used as in the above lemma. First differentiate the functional equation in the form $\zeta(1-s)=\Lambda(s) \zeta(s)$ to obtain

$$
-\zeta^{\prime}(1-s)=\Lambda^{\prime}(s) \zeta(s)+\Lambda(s) \zeta^{\prime}(s) .
$$

If $\zeta(s)=0$, then

$$
-\zeta^{\prime}(1-s)=\Lambda(s) \zeta^{\prime}(s)
$$

Now

$$
\overline{\zeta^{\prime}(s)}=\zeta^{\prime}(\bar{s}),
$$

so if $y>1,0<x<\frac{1}{2}$, and $1-s=P_{+}$, then $s=\overline{P_{-}}$. By (1), if there is a simple zero at $P_{+}$, then there is a simple zero at $P_{-}$, and vice versa.

By (1) and (2)

$$
\begin{aligned}
-\overline{\zeta^{\prime}\left(P_{-}\right)} & =-\zeta^{\prime}\left(\overline{P_{-}}\right)=\Lambda\left(P_{+}\right) \zeta^{\prime}\left(P_{+}\right), \\
-\overline{\zeta^{\prime}\left(P_{+}\right)} & =-\zeta^{\prime}\left(\overline{P_{+}}\right)=\Lambda\left(P_{-}\right) \zeta^{\prime}\left(P_{-}\right) .
\end{aligned}
$$

For any $a \in \mathbb{C}, \arg a \equiv-\arg \bar{a} \bmod 2 \pi$. Hence

$$
\begin{aligned}
\arg \zeta^{\prime}\left(P_{-}\right) & \equiv \arg \Lambda\left(P_{+}\right)+\arg \zeta^{\prime}\left(P_{+}\right) \quad \bmod 2 \pi, \\
\arg \zeta^{\prime}\left(P_{+}\right) & \equiv \arg \Lambda\left(P_{-}\right)+\arg \zeta^{\prime}\left(P_{-}\right) \bmod 2 \pi .
\end{aligned}
$$

Subtracting these equations leads to

$$
\arg \Lambda\left(P_{+}\right)-\arg \Lambda\left(P_{-}\right) \equiv 2\left[\arg \zeta^{\prime}\left(P_{-}\right)-\arg \zeta^{\prime}\left(P_{+}\right)\right] \bmod 2 \pi .
$$

By Lemma 4.2 above, $\arg \Lambda\left(P_{+}\right) \equiv \arg \Lambda\left(P_{-}\right) \bmod 2 \pi$, so

$$
\arg \zeta^{\prime}\left(P_{+}\right) \equiv \arg \zeta^{\prime}\left(P_{-}\right) \quad \bmod 2 \pi,
$$

and therefore the zeros have the same type [6]. They are never saddles, since this is a general property of the zeros of holomorphic functions.

In the proof of the theorem below, to make formulas more compact, the abreviations $c=\cos (\theta), s=\sin (\theta),\langle n\rangle=\log n$ are used. 
Theorem 4.6. In the neigborhood of any simple zero of $\zeta(z)$ on the critical line, the flow $\dot{z}=\zeta(z)$ is never that of a node or center, i.e., the flow is a focus.

Proof. Let $\rho=\frac{1}{2}+i \gamma$ be a simple zero of $\zeta(z)$ with $\gamma>0$. The proof is by contradiction, and is organized as follows. Zeta is represented in $\sigma \in(0,1)$ by an alternating series. The real and imaginary parts of $\zeta(z)$ are expressed in translated polar coordinates $r$ and $\theta$, where $z=\frac{1}{2}+i \gamma+r e^{i \theta}$. Expressions for $\dot{r}$ and $\dot{\theta}$ are derived, and the coefficient of $r$ in the right-hand side of $\dot{z}=\zeta(z)$ is evaluated. The constant term vanishes (as it should) in each case. If $w$ is a local complex variable, then the linearization of the flow should have the form $\dot{w}=(\alpha+i \beta) w$, where $\alpha$ and $\beta$ are real. Taking the limit as $r \rightarrow 0+$, if either $\alpha=0$ or $\beta=0$ we show that $\zeta^{\prime}(\rho)=0$, which is not possible. This contradiction shows that neither $\alpha$ nor $\beta$ can be zero. The result of the theorem then follows from [ 6$]$.

1. First a number of preliminary transformations to polar coordinates are made:

$$
\begin{aligned}
C & :=\frac{1}{1-2^{1-s}}, \\
\Re C & =\left(1-2^{\frac{1}{2}-r c} \cos (\langle 2\rangle(\gamma+r s))\right) / d, \\
\Im C & =2^{\frac{1}{2}-r c} \sin (\langle 2\rangle(\gamma+r s)), \text { where } \\
d & :=1+2^{1-2 r c}-2 \cdot 2^{\frac{1}{2}-r c} \cos (\langle 2\rangle(\gamma+r s)), \\
\zeta(z) & =C \times \sum_{n=1}^{\infty} \frac{(-1)^{n+1}}{n^{z}}=C \times S, \text { where } \\
\Re S & =\sum_{n=1}^{\infty}(-1)^{n+1} \frac{\cos (\langle n\rangle(\gamma+r s))}{n^{\frac{1}{2}+r c}}, \\
\Im S & =-i \sum_{n=1}^{\infty}(-1)^{n+1} \frac{\sin (\langle n\rangle(\gamma+r s))}{n^{\frac{1}{2}+r c}},
\end{aligned}
$$

$$
\Re \zeta(z)=\frac{1}{d} \sum_{n=1}^{\infty} \frac{(-1)^{n+1}}{n^{\frac{1}{2}}+r c}\left[\cos (\langle n\rangle(\gamma+r s))-2^{\frac{1}{2}-r c} \cos (\langle 2 n\rangle(\gamma+r s))\right]
$$

$$
\begin{gathered}
\Im \zeta(z)=\frac{1}{d} \sum_{n=1}^{\infty} \frac{(-1)^{n+1}}{n^{\frac{1}{2}+r c}}\left[-\sin (\langle n\rangle(\gamma+r s))+2^{\frac{1}{2}-r c} \sin (\langle 2 n\rangle(\gamma+r s))\right], \\
\frac{\dot{r}}{r}=\frac{\cos (\theta)}{r} \Re \zeta(z)+\frac{\sin (\theta)}{r} \Im \zeta(z), \\
\dot{\theta}=\frac{\cos (\theta)}{r} \Im \zeta(z)-\frac{\sin (\theta)}{r} \Re \zeta(z) .
\end{gathered}
$$

2. The values for the real and imaginary parts of $\zeta(z)$ given in (1) and (2) are then substituted in the expression (4) for $\dot{\theta}$ :

$$
\dot{\theta}=\frac{1}{r d} \sum_{n=1}^{\infty} \frac{(-1)^{n+1}}{n^{\frac{1}{2}+r c}}\left[-\sin (\theta+\langle n\rangle(\gamma+r s))+2^{\frac{1}{2}-r c} \sin (\theta+\langle 2 n\rangle(\gamma+r s))\right] .
$$


The result is then expanded as a Laurent series in $r$. Since $\zeta\left(\frac{1}{2}+i \gamma\right)=0$, the real and imaginary parts give

$$
\begin{gathered}
\sum_{n=1}^{\infty} \frac{(-1)^{n+1}}{n^{\frac{1}{2}}}[-\sin (\langle n\rangle \gamma)+\sqrt{2} \sin (\langle 2 n\rangle \gamma)]=0 \\
\sum_{n=1}^{\infty} \frac{(-1)^{n+1}}{n^{\frac{1}{2}}}[\cos (\langle n\rangle \gamma)-\sqrt{2} \cos (\langle 2 n\rangle \gamma)]=0 .
\end{gathered}
$$

Multiply the first of these equations by $\cos (\theta)$ and the second by $-\sin (\theta)$ to show that the leading coefficient of $1 / r$ in the expansion of $\dot{\theta}$ in powers of $r$ is zero:

$$
\sum_{n=1}^{\infty} \frac{(-1)^{n+1}}{n^{\frac{1}{2}}}[-\sin (\theta+\langle n\rangle \gamma)+\sqrt{2} \sin (\theta+\langle 2 n\rangle \gamma)]=0 .
$$

3. Extracting the coefficient of the constant term in the expansion and rearranging terms, we obtain

$$
\begin{aligned}
& \cos (\theta) \sum_{n=1}^{\infty}(-1)^{n+1} \frac{\langle n\rangle}{\sqrt{n}}[-\cos (\langle n\rangle \gamma)+\sqrt{2} \cos (\langle 2 n\rangle \gamma)] \\
+ & \sin (\theta) \sum_{n=1}^{\infty}(-1)^{n+1} \frac{\langle n\rangle}{\sqrt{n}}[\sin (\langle n\rangle \gamma)-\sqrt{2} \cos (\langle 2 n\rangle \gamma)] .
\end{aligned}
$$

If the point $\frac{1}{2}+i \gamma$ is a node for the flow, then $\lim _{r \rightarrow 0+} \dot{\theta}$ would vanish. But that means the expression (5) would vanish for all $\theta$, so the coefficients of $\cos (\theta)$ and $\sin (\theta)$ would vanish. Multiplying the former by -1 and the latter by $i$ and adding leads to

$$
\sum_{n=1}^{\infty}(-1)^{n+1} \frac{\langle n\rangle\left[e^{\langle n\rangle \gamma}-\sqrt{2} e^{\langle 2 n\rangle \gamma}\right]}{\sqrt{n}}=0
$$

so

$$
\sum_{n=1}^{\infty}(-1)^{n+1} \frac{\langle n\rangle}{\sqrt{n}} n^{i \gamma}\left(1-\sqrt{2} 2^{i \gamma}\right)=0
$$

so

$$
\sum_{n=1}^{\infty}(-1)^{n+1} \frac{\langle n\rangle n^{i \gamma}}{\sqrt{n}}=0
$$

By analytic continuation from $\Re z>1$, it can be seen that this series represents a value of the derivative of $S$, so $S^{\prime}\left(\frac{1}{2}-i \gamma\right)=0$. But $\zeta(z)=C(z) \times S(z)$, so therefore

$$
\zeta^{\prime}\left(\frac{1}{2}-i \gamma\right)=C\left(\frac{1}{2}-i \gamma\right) S^{\prime}\left(\frac{1}{2}-i \gamma\right)+C^{\prime}\left(\frac{1}{2}-i \gamma\right) S\left(\frac{1}{2}-i \gamma\right)=0,
$$

which is impossible, since $\frac{1}{2}-i \gamma$ is a simple zero of $\zeta(s)$. This contradiction shows that the zero of zeta is not a node for the flow.

5. The values for the real and imaginary parts of $\zeta(z)$ are next substituted in the expression (3) for $\dot{r} / r$ :

$$
\frac{\dot{r}}{r}=\frac{1}{r d} \sum_{n=1}^{\infty} \frac{(-1)^{n+1}}{n^{\frac{1}{2}+r c}}\left[\cos (\theta+\langle n\rangle(\gamma+r s))-2^{\frac{1}{2}-r c} \cos (\theta+\langle 2 n\rangle(\gamma+r s))\right] .
$$

6. As before, the leading coefficient of $1 / r$ is zero. 
7. Now assume the flow is that of a center in the neighborhood of $z=\frac{1}{2}+i \gamma$. Then $\lim _{r \rightarrow 0+} \dot{r} / r=0$. Extracting the coefficient of the constant term in the expansion of the right-hand side of (6) in powers of $r$ and simplifying in the same manner as in 3 again, we can derive the equation $\zeta^{\prime}\left(\frac{1}{2}-i \gamma\right)=0$, again giving a contradiction.

8. Since $\zeta(z)$ is holomorphic, the flow cannot be a saddle 6 . Since it is nondegenerate at $z=\frac{1}{2}+i \gamma$, it must therefore have the form of a focus.

\section{Numerical evaluation}

The Riemann zeta function is defined for complex $s=\sigma+i t$ as

$$
\zeta(s)=\sum_{j=0}^{\infty} \frac{1}{(j+1)^{s}}, \quad \Re s>1 .
$$

An equivalent expression with terms alternating in sign is

$$
\zeta(s)=\frac{1}{1-2^{1-s}} \sum_{j=0}^{\infty} \frac{(-1)^{j}}{(j+1)^{s}}, \quad \Re s>0,
$$

with the corresponding integral

$$
\zeta(s)=\frac{1}{\left(1-2^{1-s}\right) \Gamma(s)} \int_{0}^{\infty} \frac{y^{s-1}}{e^{y}+1} d y, \quad \Re s>0,
$$

and where the gamma function $\Gamma(s)$ has the integral form

$$
\Gamma(s)=\int_{0}^{\infty} y^{s-1} e^{-y} d y=\int_{0}^{\infty} \frac{y^{s-1}}{e^{y}} d y, \quad \Re s>0 .
$$

The poles of the gamma function are at the nonpositive integers $s=-n$ for $n=0,1,2, \ldots$, while $\zeta(s)$ has a simple pole at $s=1$; the trivial zeros of $\zeta(s)$ are at $s=-2 n$, with $n=1,2,3, \ldots$.

For the phase portraits we required accuracies of at least 6 significant figures in the calculation of the zeta function. There are very few nonzero values of $\zeta(s)$ published. The calculations of Haselgrove [9] are the most extensive, but only cover $\zeta\left(\frac{1}{2}+i t\right)$ and $\zeta(1+i t)$ for $0 \leq t \leq 100$ to accuracies of 6 significant figures. Here we needed values over the range $-10<\sigma<100$ and at least $-800<t<800$ to compute the phase portraits by integration of $\dot{s}=\zeta(s)$.

The literature on zeta evaluation is dominated by calculations of the critical zeros along the critical line $\zeta\left(\frac{1}{2}+i t\right)$ (because of the Riemann Hypothesis), and for this purpose normally the Riemann-Siegel formula is used together with zeta evaluation using some form of Euler-MacLaurin summation [1,2,4,16]. A source of these zeros is [16], where 100,000 zeros are listed to 8 significant figures, and the first 100 are computed to 1000 significant figures. The current limit on the number of zeros computed to sufficient accuracy to ensure that they lie on the critical line is 150,000,001 [3], [13. An independent method is that of Galway 10], which uses saddle-point integration and achieves high precision (greater than 1000 significant figures). Each of these is rather specialized, with much detail required, and we turned to a more transparent method. Another independent method is that of convergence acceleration of alternating series, as discussed in [11]. This promises to be both efficient and powerful. Further approaches exist as algorithms [14], [15], [16], but perhaps not yet as available code. 
5.1. Choice of method and accuracy. We decided to use the Chebyshev method of P. Borwein [5]. He presents two versions of a generic method, the Chebyshev version and a binomial version. The binomial method requires twice as many terms (and hence numerical operations) for the same theoretical accuracy.

The approach taken is to find weighting coefficients $b_{j}$ which decrease to zero and which smooth the finite sum for zeta (compare (1))

$$
\zeta(s)=\frac{1}{1-2^{1-s}} \sum_{j=0}^{n-1} \frac{(-1)^{j} b_{j}}{(j+1)^{s}}+\gamma_{n}(s), \quad \Re s>0 .
$$

We note that our $b_{j}$ is $-c_{j} / d_{n}$ in Borwein's notation, $d_{n}$ being a normalizing factor. When $n$ terms are used (in practice $n=402$ allows us to compute $\zeta(s)$ as far as $t<680$ to 10 significant figures accuracy) we have the approximate sum and an error term $\gamma_{n}(s)$ as given.

The smoothing coefficients $b_{j}$ are written in terms of the coefficients of the $n^{\text {th }}$ order Chebyshev polynomial shifted to $[0,1]$ as

$$
b_{j}=1-\frac{d_{j}}{d_{n}},
$$

where the $d_{j}$ are given by

$$
d_{j}=n \sum_{i=0}^{j} \frac{(n+i-1) ! 4^{i}}{(n-i) !(2 i) !} .
$$

For the error term, $\gamma_{n}(s)$, Borwein gives

$$
\left|\gamma_{n}(s)\right| \leq \frac{2}{5.83^{n}} \frac{1}{\left|\left(1-2^{1-s}\right) \Gamma(s)\right|}, \quad \Re s>\frac{1}{2} .
$$

Since $\Gamma(s)$ is readily computable (see below), we need no further approximation to the bound. We did not in fact find it helpful, and so we turned to empirical means to assess the error.

A program written in MATLAB served as the basis for our code. Godfrey [11] programmed the Chebyshev method and, most importantly, he numerically investigated the Lanczos method of computing the gamma function on the complex half-plane and found an optimum coefficient set for double precision (approximately 15 significant figures) using 15 terms [11. It is available in Godfrey's MATLAB suite as part of the zeta function code. In particular, values of the gamma function are needed for the reflection formula used when $\Re s=\sigma<\frac{1}{2}$ :

$$
\zeta(s)=2(2 \pi)^{s-1} \sin \left(\frac{1}{2} \pi s\right) \Gamma(1-s) \zeta(1-s) .
$$

Values of the zeta function were computed at the 8-significant-figure zeros of Odlyzko using 402 terms; then inverse interpolation on the (real) Riemann-Siegel function $Z(t)$ enabled the accuracy of the zeros to be increased to better than $10^{-10}$ over the range $0<t<682$, which contains the first 402 zeros on the critical line.

The graphs in Figure 9 illustrate the reduction in accuracy with the decrease in the number of terms.

The symbolic programs Mathematica, Maple, etc., allowed checks of selected values at substantially higher precision. 

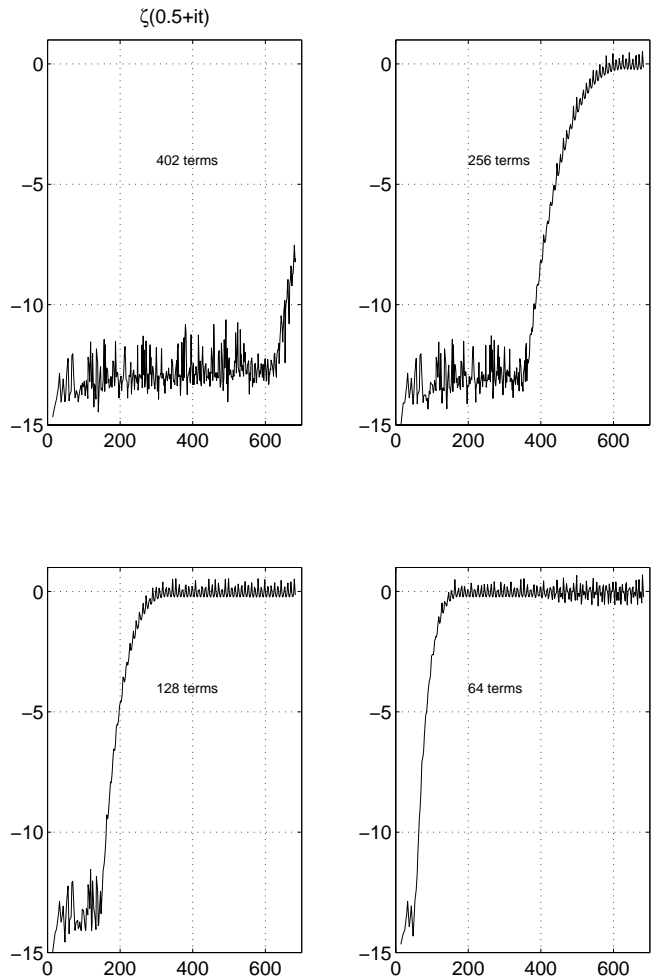

Figure 9. Accuracy of the Chebyshev method in computing zeros of $s=0.5+i t$. The ordinate is $\log _{10}(|\zeta|)$, while the abscissa is $t$

5.2. Conclusion. Mathematica was found to give values for $\zeta(s)$ in the critical strip in agreement with those found using the MATLAB programs implementing the Chebyshev method. It proved to be more convenient to produce some of the plots included in this paper using Mathematica, and some using MATLAB. The original plots, initiating this investigation, were produced using programs written in Java. These have the advantage of enabling the phase plane to be investigated interactively.

\section{Conjectures}

1. With four exceptions, every separatrix is unbounded.

2. There exist an infinite number of sources and an infinite number of sinks on the critical line.

3. The holomorphic flow of the zeta function has no periodic orbits.

Definition 6.1. We say the trajectory $\gamma(\tau)$, which is a solution of $\dot{s}=\zeta(s)$, crosses the critical strip positively if there exist $\tau_{1}, \tau_{2}$ with $\tau_{1}<\tau_{2}$ and such that $\Re \gamma\left(\tau_{1}\right)=0$ and $\Re \gamma\left(\tau_{2}\right)=1$.

4. There exist an infinite number of trajectories which cross the critical strip positively.

5. If $\left(\beta_{n}\right)$ is the sequence of distances between the separatrices going to infinity in the $\mathrm{E}$ direction, then $\lim _{n \rightarrow \infty} \beta_{n}$ exists and has the value 0 . 
6. The separatrices going to infinity in the $\mathrm{E}$ direction are asymptotic to the horizontal lines at height $t$ through the critical points of the Riemann-Siegel function $Z(t)$.

\section{ACKNOWLEDGMENTS}

The support of the University of Waikato Department of Mathematics and of Pat Gallagher of Columbia University are warmly acknowledged. The assistance of Francis Kuo with the Java phase portrait plotting program development, and valuable insights provided by A. Steyn-Ross, are also warmly acknowledged.

\section{REFERENCES}

[1] T. Apostol, Introduction to Analytic Number Theory, Springer-Verlag, 1976. MR 55:7892

[2] M. V. Berry and J. P. Keating, A new asymptotic representation of $\zeta\left(\frac{1}{2}+i t\right)$ and quantum spectral determinants, Proc. Roy. Soc. London A 437 (1992), 151-173. MR 93j:11057

[3] R. P. Brent, On the zeros of the Riemann zeta function in the critical strip, Math. Comp. 33 (1979), 1361-1372. MR 80g:11033

[4] J. M. Borwein, D. M. Bradley, and R. E. Crandall, Computational strategies for the Riemann zeta function, J. Comp. Appl. Math. 121 (2000), 247-296. MR 2001h:11110]

[5] P. Borwein, An efficient algorithm for the Riemann zeta function, Constructive, experimental and nonlinear analysis (Limoges, 1999), CMS Conf. Proc. 27 (2000), 29-34. MR 2001f:11143

[6] K. A. Broughan, Holomorphic flows on simply connected domains have no limit cycle, Meccanica 38(6) (2003), 699-709.

[7] K. A. Broughan, http://www.math.waikato.ac.nz/ kab, Web site for zeta phase portraits and data for the zeros encoding.

[8] E. A. Coddington and N. Levinson, Theory of Ordinary Differential Equations, McGraw-Hill, 1955. MR 16:1022b

[9] C. B. Haselgrove, Tables of the Riemann Zeta Function, Roy. Soc. Math. Tables 6 (1960), 2-22. MR 22:8679

[10] W. F. Galway, Computing the Riemann zeta function by numerical quadrature, Dynamical, spectral and arithmetic zeta functions, (San Antonio, Texas, 1999), Contemp. Math. 290, 81-91. MR 2002i:11131

[11] P. Godfrey, An efficient algorithm for the Riemann zeta function, http://www.mathworks. com/support/ftp zeta.m, etan.m (2000)

[12] U. D. Jentschura, P. J. Mohr, G. Soff, E. J. Weniger, Convergence acceleration via combined nonlinesr-condensation transformations, Computer Physics Comm. 116 (1999), 28-54.

[13] J. van de Lune, H. J. J. te Riele and D. T. Winter, On the zeros of the Riemann zeta function in the critical strip, IV, Math. Comp. 46 (1986), 667-681. MR 87e:11102

[14] A. M. Odlyzko and A. Schönhage, Fast algorithms for multiple evaluations of the Riemann zeta function, Trans. Amer. Math. Soc. 309 (1988), 797-809. MR 89j:11083

[15] A. M. Odlyzko, Analytic Computation in Number Theory, Proc. Symp. Appl. Math. 48 (1994), 451-463. MR 96a:11146

[16] A. M. Odlyzko, The first 100,000 zeros of the Riemann zeta function, http://www. research.att.com/ amo, (1997).

[17] S. J. Patterson, An introduction to the theory of the Riemann Zeta-function, Cambridge, 1988. MR 89d:11072

[18] L. Perko, Differential Equations and Dynamical Systems, Second Edition, Springer, 1996. MR 97g:34002

[19] A. Speiser, Geometrisches zur Riemannschen Zetafunktion, Math. Annalen 110 (1934), 514521.

[20] E.C. Titchmarsh and D.R. Heath-Brown, The theory of the Riemann zeta-function, Oxford, Second Edition, 1986. MR 88c:11049

University of Waikato, Hamilton, New Zealand

E-mail address: kab@waikato.ac.nz

University of Waikato, Hamilton, New Zealand

E-mail address: arbus@waikato.ac.nz 